\title{
A combined analysis of 3D weak lensing, Lyman- $\alpha$ forest and WMAP year three data
}

\author{
J Lesgourgues ${ }^{1}, \mathrm{M} \mathrm{Viel}{ }^{2,3}, \mathrm{M}$ G Haehnelt ${ }^{4}$ and R Massey ${ }^{5}$ \\ ${ }^{1}$ LAPTH, Université de Savoie and CNRS (UMR5108), BP110, F-74941 \\ Annecy-le-vieux Cedex, France \\ 2 INAF-Osservatorio Astronomico di Trieste, Via G.B. Tiepolo 11, \\ I-34131 Trieste, Italy \\ ${ }^{3}$ INFN/National Institute for Nuclear Physics, Via Valerio 2, I-34127 Trieste, \\ Italy \\ ${ }^{4}$ Institute of Astronomy, Madingley Road, Cambridge CB3 0HA, UK \\ ${ }^{5}$ California Institute of Technology, 1200 East California Boulevard, Pasadena, \\ CA 91125, USA \\ E-mail: lesgourg@lapp.in2p3.fr, viel@oats.inaf.it, haehnelt@ast.cam.ac.uk and \\ rjm@astro.caltech.edu
}

Received 7 May 2007

Accepted 8 October 2007

Published 9 November 2007

Online at stacks.iop.org/JCAP $/ 2007 / \mathrm{i}=11 / \mathrm{a}=008$

doi:10.1088/1475-7516/2007/11/008

\begin{abstract}
We present constraints on the amplitude and shape of the matter power spectrum and the density of dark matter within the framework of a standard $\Lambda \mathrm{CDM}$ model. We use a Markov chain Monte Carlo approach to combine independent measurements of the three-dimensional weak gravitational lensing shear field by the COSMOS survey, of low- and high-resolution Lyman- $\alpha$ $(\operatorname{Ly} \alpha)$ forest flux power spectrum by SDSS and LUQAS, and of cosmic microwave background temperature and polarization anisotropies by WMAP. We note good agreement between the amplitude of the matter power spectrum on intermediate and small scales as inferred from Ly $\alpha$ forest and lensing data. The Ly $\alpha$ forest data help to break the $\sigma_{8}-\Omega_{0 \mathrm{~m}}$ degeneracy characteristic of weak lensing results, yielding $\sigma_{8}=0.876 \pm 0.048$ for COSMOS plus Ly $\alpha$ SDSS data. This is somewhat larger than the value preferred by the WMAP year three CMB data. Combining all three data sets significantly tightens the constraints on $\sigma_{8}$, the spectral index of primordial density fluctuation $n_{\mathrm{s}}$, a possible running of the spectral index $n_{\text {run }}$ and the matter density $\Omega_{0 \mathrm{~m}}$. Assuming no running, the joint constraints
\end{abstract}


for COSMOS, SDSS and WMAP are $\sigma_{8}=0.800 \pm 0.023, n_{\mathrm{s}}=0.971 \pm 0.011$, $\Omega_{0 \mathrm{~m}}=0.247 \pm 0.016$ (1- $\sigma$ error bars).

Keywords: CMBR experiments, classical tests of cosmology, gravitational lensing, power spectrum

ArXiv ePrint: 0705.0533

\section{Contents}

1. Introduction 2

2. The data sets 3

2.1. Ly $\alpha$ forest data . . . . . . . . . . . . . . . . . . . . . . 3

2.2. Weak lensing data . . . . . . . . . . . . . . . . . . 4

3. Results

4. Conclusions

Acknowledgments

References

\section{Introduction}

The recent measurement of the cosmic microwave background (CMB) anisotropies obtained by the WMAP satellite $[7,11,12]$ has considerably tightened the error bars on the cosmological parameters that describe the standard $\Lambda$ CDM model of structure formation. The constraining power of the WMAP year three measurements alone is already large. Cosmological parameter extraction nevertheless benefits from a combination of the CMB data with measurements of the matter power spectrum on smaller scales and at different redshifts. The various observables suffer from very different systematic and statistical errors, and a combined analysis can break degeneracies inherent to individual measurements.

We concentrate here on comparing and combining the CMB data with weak gravitational lensing and $\operatorname{Ly} \alpha$ forest data. The $\operatorname{Ly} \alpha$ forest due to the absorption produced by neutral hydrogen along the line-of-sight to distant quasars (QSOs) allows us to measure the matter power spectrum on scales that range from a few to tens of comoving Mpc at $z=2-4$ (e.g. [2]). 'Cosmic shear' measurements of the distortion induced in distant galaxy images by weak gravitational lensing around foreground structures map the mass distribution at similar scales but smaller redshift, $z=0-1.5$. Both methods probe the matter power spectrum on smaller scales than CMB anisotropies observed by WMAP, and offer a more direct measurement of the quantity $\sigma_{8}$, the rms of the density fluctuations in spheres of radii 8 comoving $h^{-1} \mathrm{Mpc}$. Furthermore, the small scale matter power spectrum inferred from the Ly $\alpha$ forest data puts strong limits on the mass of warm dark matter candidates and on isocurvature perturbations (e.g. [1, 18], [22]-[24]).

Viel et al [33] and Seljak et al [22] have recently presented independent analyses of CMB data combined with $\operatorname{Ly} \alpha$ forest data from separate, state-of-the-art QSO samples: the LUQAS sample of high-resolution high-quality VLT-UVES spectra $[9,16,34]$ and a 
large sample of SDSS QSO spectra [10]. Despite the very different data sets and the use of different analysis techniques, both groups found similar results, suggesting a value for $\sigma_{8} \sim 0.9$ larger than that extrapolated from the CMB data alone $\left(\sigma_{8}=0.76 \pm 0.05\right)$. For the case of the SDSS Ly $\alpha$ forest data there appears to be a moderate tension between the two data sets at the $2 \sigma$ level [22]. For the LUQAS data the errors are about a factor two larger and the difference is not statistically significant [33]. Reference [8] obtained very similar results with a further $\operatorname{Ly} \alpha$ forest data set.

The moderate but notable tension between the amplitude of the matter power spectrum inferred from the SDSS Ly $\alpha$ forest data with the WMAP year three results suggests that measurements of the amplitude of the small and intermediate scale matter power spectrum are still somewhat uncertain. Further comparison with a third, completely independent technique should be very useful in this context. We exploit the tomographic weak gravitational lensing analysis used to map the three-dimensional distribution of mass in the Hubble Space Telescope COSMOS survey [27,28]. The clustering signal in that distribution also corresponds to a relatively large value of $\sigma_{8}=0.95_{-0.075}^{+0.093}$ (when the matter fraction $\Omega_{0 \mathrm{~m}}$ is chosen to be the WMAP maximum likelihood value). A recent combination of two-dimensional, ground-based cosmic shear surveys into the 100 square degree Weak Lensing Survey gives a comparable although slightly smaller amplitude of the matter power spectrum $\sigma_{8}=0.84 \pm 0.07$ (for the best fit $\Omega_{0 \mathrm{~m}}$ WMAP value).

In this paper, we investigate in detail to what extent the Ly $\alpha$ forest and the cosmic shear data of Massey et al (2007) are consistent, and present new constraints on the value of cosmological parameters obtained from various combinations of cosmic shear, Ly $\alpha$ forest and CMB data (for the latter we limit ourselves to the WMAP year three determination of temperature/polarization anisotropies [12]). We use the Boltzmann code CAMB [31] for computing the linear matter power spectrum and applying relevant nonlinear corrections [30]. The multi-dimensional parameter space is explored with Monte Carlo Markov chains (MCMC) using the public code Cosmomc [21].

\section{The data sets}

\subsection{Ly $\alpha$ forest data}

We have used two Ly $\alpha$ forest data sets: (i) the high-resolution QSO absorption spectra presented in Viel, Haehnelt and Springel (VHS) [16] and in [19,33], consisting of the LUQAS sample [9] and the reanalyzed Croft et al (C02) [2] data; (ii) the SDSS Ly $\alpha$ forest sample presented in [10] (M05). The SDSS Ly $\alpha$ forest data set consists of 3035 QSO spectra with low resolution $(R \sim 2000)$ and low signal-to-noise $(<10$ per pixel $)$ spanning a wide range of redshifts $(z=2.2-4.2)$, while the LUQAS and the C02 samples contain mainly 57 high-resolution $(R \sim 45000)$, high signal-to-noise ( $>50$ per pixel) QSO spectra with median redshifts of $z=2.125$ and 2.72 , respectively.

The flux power spectrum of the Ly $\alpha$ forest is the quantity which is observed and needs to be modeled at the per cent or sub-per cent level using accurate numerical simulations that incorporate the relevant cosmological and astrophysical processes. M05 modeled the flux power spectrum using a large number of hydro particle mesh (HPM) simulations $[4,17]$, calibrated with full hydrodynamical simulations. Instead, the VHS analysis significantly improved the effective bias method developed by C02 (see [3] and [20] for a critical assessment of the errors involved), by using a grid of full hydrodynamical simulations run with the Tree-SPH code GADGET-2 $[13,14]$ to infer the linear matter 
power spectrum. Finally, Viel and Haehnelt [15] performed an independent analysis of the SDSS Ly $\alpha$ forest data, and used a Taylor expansion of the flux power spectrum around best-fitting values based on full hydrodynamical simulations to model the dependence of the flux power on cosmological and astrophysical parameters. This analysis was performed directly on the flux power spectrum and took thus full advantage of each data point, for a wider range of parameters than just the amplitude and slope constrained by the SDSS analysis. One should however keep in mind that the Taylor expansion approach is likely to underestimate the errors far from best-fit values. However, as soon as the SDSS Lyman- $\alpha$ data are combined with either COSMOS or WMAP, large departures from the best-fit model are forbidden and the Taylor method is accurate.

In this paper, we will use either the data set of VHS, based on high-resolution QSO spectra and denoted 'Lya VHS'; or the results of [15] using low-resolution SDSS spectra and a Taylor expansion, denoted 'Lya SDSS-d' (where-d refers to 'derivatives', since this method is based on the derivatives of the flux power spectrum.

Our COSMOMC module lya.f90 comparing the linear dark matter power with Lya VHS data has been incorporated into the latest publicly available version of COSMOMc [21]. The Lya VHS power spectrum consists of estimates of the linear dark matter power spectrum at nine values in the wavenumber space $k$ at $z=2.125$ and nine values at $z=2.72$, in the range $0.003<k\left(\mathrm{~s} \mathrm{~km}^{-1}\right)<0.03$. The estimate of the uncertainty of the overall amplitude of the matter power spectrum is $29 \%$. This estimate takes into account possible systematic and statistical errors (see the relevant tables of VHS for a detailed discussion). The code assigns a Gaussian prior to the corresponding nuisance parameter and marginalizes over it. For the Lya SDSS-d analysis, we used the COSMOMC module described in [33], which involves 21 nuisance parameters characterizing a wide range of astrophysical and noise-related systematic uncertainties. In the following results these parameters are always marginalized out.

\subsection{Weak lensing data}

The Hubble Space Telescope COSMOS survey covers a contiguous area of 1.64 square degrees on the sky. In this high-resolution, space-based data, the shapes of 234370 distant galaxies were measured, with a median $F 814 W_{A B}$-band magnitude of $24.6[26,27]$.

A crucial addition to this survey has been the acquisition of ground-based imaging in 15 extra bands. Photometric redshift estimation for each galaxy allows a fully 3D exploitation of the signal, in which the power spectrum can be independently measured at different redshifts and physical scales. In a 2D analysis, these would have been projected together, resulting in a loss of discriminatory power on cosmological parameters of a factor of $3-5[25,27]$. BPZ photometric redshift estimation software achieved $68 \%$ confidence limits of $0.03(1+z)$ on each galaxy to $z \sim 1.4$ and $I_{F 814 W}=24$ [29]. The galaxy catalog has a median photometric redshift of $z_{\text {phot }}=1.26$, and has been split into three redshift bins for this analysis: $z_{\text {phot }}=0.1-1, z_{\text {phot }}=1-1.4$ and $z_{\text {phot }}=1.4-3$, which divide the number of sources almost evenly.

In each redshift bin, the 'cosmic shear' two-point correlation functions $C_{1,2}(\theta)$ have been measured on angular scales 0.1-40 arcmin [27]. Error estimates for these measurements include: statistical errors, calculated from the internal distribution of shear estimators; cosmic (sample) variance, calculated from the variation in the signal between separate quadrants of the COSMOS field; and finally systematic errors, which include the 
potential bias in shear calibration (overall bias and relative bias between redshift bins), errors due to catastrophic photometric redshift failures, and errors due to binning.

In Massey et al [27], the constraints on cosmological parameters were derived as follows. For a three-dimensional grid of models spanning variations of $\Omega_{0 \mathrm{~m}}$ from 0.05 to $1.1, \sigma_{8}$ from 0.35 to 1.4 and the power spectrum shape parameter $\Gamma$ from 0.13 to 0.33, the linear power spectra were obtained from the fitting formula of BBKS [35], and corrected for non-linear evolution using HALOFIT [30]. For each model, the data likelihood was computed taking only statistical errors into account. Then, the threedimensional likelihood distribution was integrated in order to marginalize over $\Gamma$ and to obtain confidence contours for $\left(\Omega_{0 \mathrm{~m}}, \sigma_{8}\right)$. The best-constrained combination of these parameters was found to be $\sigma_{8}\left(\Omega_{0 \mathrm{~m}} / 0.3\right)^{0.44}$. Final bounds on this quantity were obtained by adding systematic error linearly.

In this work, we wrote a COSMOMC module for COSMOS data (downloadable at http://www.astro.caltech.edu/ rjm/cosmos/cosmomc/) which offers various advantages with respect to the original analysis: the linear power spectrum is computed by CAMB, more free parameters can easily be implemented in the analysis (like the tilt $n_{S}$ ), and the systematic errors can be accounted more accurately by introducing various nuisance parameters. The module computes the shear correlation functions for any cosmological model explored by the Markov chains (including corrections to the linear power spectrum obtained with the HALOFIT $\operatorname{code}^{6}$ ) and compares with the tomographic results from [27], neglecting the cross-correlation between redshift slices. As shown in figure 8 of [27], there are measurements on six angular scales, in each of three redshift bins. Since there are two correlation functions available for shear, the number of data points sums up to 36 . The $36 \times 36$ covariance matrix is presented in figure 9 of [27], and we used its inverse when calculating the likelihood. We modeled the systematic errors of the lensing data described in detail in [27] by three nuisance parameters, over which our final results are marginalized. A blind analysis of simulated HST images suggests a potential $6 \%$ uncertainty in the overall calibration of the shear measurement [26], for which we account with a parameter $A$ with Gaussian prior. We further introduce a parameter $B$ to account for a $5 \%$ relative calibration uncertainty between the shear measured from galaxies in our high and low redshift bins. Although not seen in the simulated HST simulations of [26], more comprehensive tests on a larger set of simulated ground-based images $[5,6]$ reveal the potential for the shear to be underestimated in faint or small galaxies. The opposite effect has not been recorded. $B$ is thus assigned a one-sided Gaussian prior. Finally, a potential $10 \%$ intrusion of low redshift galaxies into the high redshift bin due to catastrophic photometric redshift errors is modeled with a third nuisance parameter, $C$. Priors on the photometric redshifts were designed to ensure that the effect on lensing observables of known types of catastrophic failure are easily modeled. The failures can only dilute the signal in the high redshift bin, and again we use a one-sided Gaussian prior. In summary, the data points $C_{1,2}(\theta)$ are multiplied by

$$
\begin{aligned}
& (A / B)^{2} \quad(\text { low-z }) \\
& A^{2} \quad(\text { medium-z) } \\
& (A B)^{2} / C \quad \text { (high-z) }
\end{aligned}
$$

\footnotetext{
${ }^{6}$ The authors of [27] checked that using Peacock and Dodds fitting functions instead of HALOFIT only affects the result on $\sigma_{8}$ by $\sim 5 \%$. Note also that on these scales, one might investigate the impact of baryon physics on the matter power spectrum [32], although this effect is still uncertain.
} 
with priors on $A, B$ and $C$ peaking at one with $B \geq 1, C \geq 1, \sigma_{A}=0.06, \sigma_{B}=0.05$ and $\sigma_{C}=0.10$.

\section{Results}

For our MCMC analysis we have assumed a minimal flat $\Lambda$ CDM model, with no tensor contribution. We vary the following cosmological parameters with top-hat priors: dark matter density $\Omega_{0 c} h^{2} \in[0.01,0.99]$, baryon density $\Omega_{0 b} h^{2} \in[0.005,0.1]$, primordial spectral index $n_{s} \in[0.5,1.5]$, primordial amplitude $\log \left[10^{10} A_{s}\right] \in[2.7,4.0]$ and angular diameter of the sound horizon at last scattering $\theta \in[0.5,10]$. When CMB data are included, we also vary the optical depth to reionization $\tau \in[0.01,0.8]$. For part of the MCMC analysis, we have finally considered a running of the spectral index, $n_{\text {run }} \in[-0.5,0.5]$. From the parameters above the MCMC code derives the reduced Hubble parameter $h$, the matter fraction $\Omega_{0 \mathrm{~m}}$ and $\sigma_{8}$ : so, these parameters have non-flat priors and the corresponding bounds must be interpreted with some care. In addition, COSMOMC imposes a weak prior on the Hubble parameter: $h \in[0.4,1.0]$. In each case and for the purpose of comparing with [27], we also compute the combination $\sigma_{8}\left(\Omega_{0 \mathrm{~m}} / 0.3\right)^{0.44}$, which is best probed by the COSMOS lensing data.

We ran first an MCMC analysis of the COSMOS WL data alone. We recall that this analysis differs from that in Massey et al [27] in four ways: the methodology (MCMC with flat priors on the cosmological parameters instead of maximum likelihood), the introduction of one extra independent parameter $n_{\mathrm{s}}$, the fact that we compute the exact linear matter power spectra numerically, and the treatment of systematic errors. In [27], the analysis performed with statistical errors only led to the bounds $\sigma_{8}\left(\Omega_{0 \mathrm{~m}} / 0.3\right)^{0.44}=$ $0.866 \pm 0.033$ ( $68 \%$ confidence level (C.L.)), which extend to $\sigma_{8}\left(\Omega_{0 \mathrm{~m}} / 0.3\right)^{0.44}=0.866_{-0.068}^{+0.085}$ when systematic errors are added linearly. Here we first performed an MCMC analysis excluding the systematic errors, and obtained $\sigma_{8}\left(\Omega_{0 \mathrm{~m}} / 0.3\right)^{0.44}=0.83 \pm 0.06$. The increased error bar with respect to [27] is presumably due to the inclusion of an arbitrary spectral index $n_{\mathrm{s}}$ in the analysis, which opens new parameter degeneracies. The mean value of Massey et al (2007) (0.866) is perfectly consistent with this value. We then incorporated systematic errors as described in the last section and found

$$
\sigma_{8}\left(\Omega_{0 \mathrm{~m}} / 0.3\right)^{0.44}=0.814 \pm 0.074 \quad(68 \% \text { C.L. }) .
$$

Note that because of non-linear corrections to the matter power spectrum, the $\sigma_{8}$ parameter cannot be viewed as a simple calibration parameter for the theoretical correlation functions. A change in $\sigma_{8}$ changes both the amplitude and the shape of the shear correlation functions in a non-trivial way. As a consequence, the impact of systematic errors on the determination of the (linear theory) parameter $\sigma_{8}\left(\Omega_{0 \mathrm{~m}} / 0.3\right)^{0.44}$ is found to be smaller than the data calibration uncertainty itself. The $68 \%$ confidence limits on each parameter are presented in the first column of table 1 . We also show the joint $68 \%$ and $95 \%$ confidence contours in $\sigma_{8}-\Omega_{0 \mathrm{~m}}$ and $\sigma_{8}-n_{\mathrm{s}}$ space in figure 1 (yellow).

For comparison, we also show in figure 1 the $\sigma_{8}-\Omega_{0 \mathrm{~m}}$ and $\sigma_{8}-n_{\mathrm{s}}$ confidence regions for each data set separately. The COSMOS data are compatible with WMAP, since the contours have some overlap even at the $68 \%$ level. In the $\sigma_{8}-\Omega_{0 \mathrm{~m}}$ space, the COSMOS and WMAP contours appear as almost orthogonal, and the overlap clearly suggests that the WL data prefer the highest $\sigma_{8}$ values allowed by WMAP. The COSMOS data are 

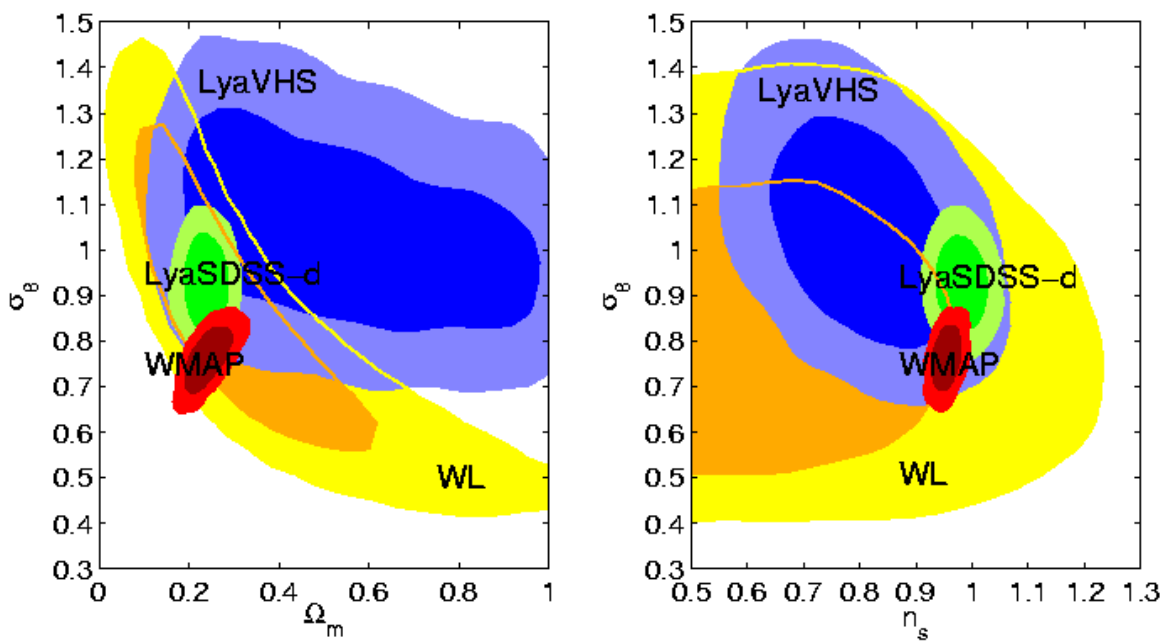

Figure 1. $1-\sigma$ and $2-\sigma$ contours of the marginalized likelihood in the $\sigma_{8}-\Omega_{0 \mathrm{~m}}$ plane (left) and $\sigma_{8}-n_{\mathrm{s}}$ plane (right) for each data set separately: WMAP (red), weak lensing (orange), Ly $\alpha$ forest from VHS [16] (blue) and from SDSS as analyzed by [15] (green).

Table 1. Summary of the constraints on $\sigma_{8}, n_{\mathrm{s}}, \Omega_{0 \mathrm{~m}}, h$ and $\tau$, for the minimal six-parameter $\Lambda C D M$ model and each data set. Since this is a Bayesian analysis, the bounds depend on our choice of priors; our top-hat priors are described at the beginning of section 3 (in particular, we impose a weak $h$ prior: $0.4<h<1.0$ ). The quoted values are either the mean and $68 \%$ C.L. error, or only the $68 \%$ C.L. upper/lower limit when a parameter is not bounded on both sides within the prior range.

\begin{tabular}{lccll}
\hline & WL & Ly $\alpha$ VHS & Ly $\alpha$ SDSS-d & WMAP3 \\
\hline$\sigma_{8}$ & $0.85 \pm 0.22$ & $1.04 \pm 0.16$ & $0.926 \pm 0.066$ & $0.762 \pm 0.046$ \\
$n_{\mathrm{s}}$ & $<0.94$ & $0.80 \pm 0.10$ & $0.982 \pm 0.028$ & $0.955 \pm 0.016$ \\
$\Omega_{0 \mathrm{~m}}$ & $0.34 \pm 0.19$ & $0.55 \pm 0.26$ & $0.238 \pm 0.030$ & $0.243 \pm 0.032$ \\
$h$ & $>0.71$ & $>0.63$ & $0.710 \pm 0.071$ & $0.729 \pm 0.030$ \\
$\tau$ & - & - & - & $<0.104$ \\
\hline
\end{tabular}

also compatible with the different $\operatorname{Ly} \alpha$ data sets, with again an overlap at the $68 \%$ level. Finally, the WMAP and Ly $\alpha$ contours overlap only at the $2 \sigma$ level, as expected from previous works $[22,33]$.

The two panels in figure 1 provide a good illustration of the advantages of combining various data sets. For each type of experiment, $\sigma_{8}$ and $\Omega_{0 \mathrm{~m}}$ are clearly correlated, but the direction of the degeneracy is different for CMB, WL and Ly $\alpha$ data. There are various reasons for the difference between the direction of correlation associated with the WL and Ly $\alpha$ data: first, the raw Ly $\alpha$ data are in units of $\mathrm{s} \mathrm{km}^{-1}$ (since the power spectrum is measured in velocity space), and the rescaling to units of $\mathrm{h} \mathrm{Mpc}^{-1}$ depends on $\Omega_{0 \mathrm{~m}}$; second, the data probe different redshifts, and the ratio between the power spectrum today (when $\sigma_{8}$ is defined) and at a given redshift depends on $\Omega_{0 \mathrm{~m}}$; third, the slope of the matter power spectrum depends on $\Omega_{0 \mathrm{~m}}$. This explains the 'banana shapes' in the upper left panels, with different orientations. 
A combined analysis of 3D weak lensing, Lyman- $\alpha$ forest and WMAP year three data

Table 2. Same as table 1 for the combination of weak lensing data with each other data set.

\begin{tabular}{llll}
\hline & WL + WMAP3 & WL + Ly $\alpha$ VHS & WL + Ly $\alpha$ SDSS-d \\
\hline$\sigma_{8}$ & $0.802 \pm 0.034$ & $0.98 \pm 0.19$ & $0.876 \pm 0.048$ \\
$n_{\mathrm{s}}$ & $0.958 \pm 0.016$ & $0.88 \pm 0.11$ & $0.962 \pm 0.034$ \\
$\Omega_{0 \mathrm{~m}}$ & $0.269 \pm 0.026$ & $0.25 \pm 0.12$ & $0.232 \pm 0.028$ \\
$h$ & $0.708 \pm 0.023$ & $>0.79$ & $0.773 \pm 0.089$ \\
$\tau$ & $<0.103$ & - & - \\
\hline
\end{tabular}

Table 3. Same as table 1 for the combination of CMB, weak lensing and Ly $\alpha$ forest data. The quoted values are the mean and $68 \%$ confidence limits.

\begin{tabular}{lll}
\hline & WL + WMAP3 + Ly $\alpha$ VHS & WL + WMAP3 + Ly $\alpha$ SDSS-d \\
\hline$\sigma_{8}$ & $0.822 \pm 0.032$ & $0.800 \pm 0.023$ \\
$n_{\mathrm{s}}$ & $0.960 \pm 0.016$ & $0.971 \pm 0.011$ \\
$\Omega_{0 \mathrm{~m}}$ & $0.282 \pm 0.026$ & $0.247 \pm 0.016$ \\
$h$ & $0.700 \pm 0.022$ & $0.730 \pm 0.016$ \\
$\tau$ & $0.094 \pm 0.028$ & $0.109 \pm 0.026$ \\
\hline
\end{tabular}

We do not find any significant correlation between $\sigma_{8}$ and $n_{s}$ (upper right panel in figure 1). For the WL and Ly $\alpha$ data, this is due to the fact that these experiments directly probe power on the scale at which $\sigma_{8}$ is defined (if this was not the case, the amplitude of the WL and Ly $\alpha$ experimental points would constrain a combination of $\sigma_{8}$ and $n_{\mathrm{s}}$ ).

We then ran some Markov chains for various combinations of different data sets and we show the results in tables 2, 3 and figure 2. Combining the data sets significantly tightens the constraints. Most noteworthy is that there remains a (moderate) strain between the inferred value of $\sigma_{8}$ between that inferred from WMAP alone and that inferred from the lensing and Ly $\alpha$ forest data. The constraint on $\sigma_{8}$ from COSMOS + Ly $\alpha$ VHS $\left(\sigma_{8}=0.98 \pm 0.19\right)$ and from COSMOS + Ly $\alpha$ SDSS-d $\left(\sigma_{8}=0.876 \pm 0.048\right)$ are compatible with the WMAP best-fit value $\left(\sigma_{8}=0.762\right)$ respectively at the 1.1- $\sigma$ and $2.4-\sigma$ level. Note that the SDSS-d contours are based on extrapolations using a Taylor expansion of the flux power spectrum around a best-fit model and are likely to underestimate the error for parameters far from the best fit model. However, when this data set is used in combination with WL and/or WMAP data, the $68 \%$ and $95 \%$ C.L. contours remain in a small region where the Taylor expansion is accurate. The main results of this work are the $68 \%$ confidence limits for the combined analysis of CMB, weak lensing and Ly $\alpha$ forest data: WMAP + COSMOS + Ly $\alpha$ VHS, $\sigma_{8}=0.822 \pm 0.032 ;$ WMAP + COSMOS + Ly $\alpha$ SDSS-d, $\sigma_{8}=0.800 \pm 0.023$.

Finally, we performed a further MCMC analysis with an extended $\Lambda$ CDM model with on extra parameter, a running of the spectral index $n_{\text {run. }}$. The results are summarized in table 4. In this case, the tilt $n_{\mathrm{s}}$ is defined at the pivot scale $k_{0}=0.01 \mathrm{Mpc}^{-1}$ (when WL and Ly $\alpha$ data are included, the pivot value $k_{0}=0.002 \mathrm{Mpc}^{-1}$ adopted in the WMAP3 paper [12] is too small with respect to the median scale of the full data set). The choice of a given pivot scale is irrelevant for the definition of $n_{\text {run }}$. WMAP alone is compatible with a rather large negative running, $n_{\text {run }}=-0.055 \pm 0.03$ (68\% C.L.) which 
A combined analysis of 3D weak lensing, Lyman- $\alpha$ forest and WMAP year three data
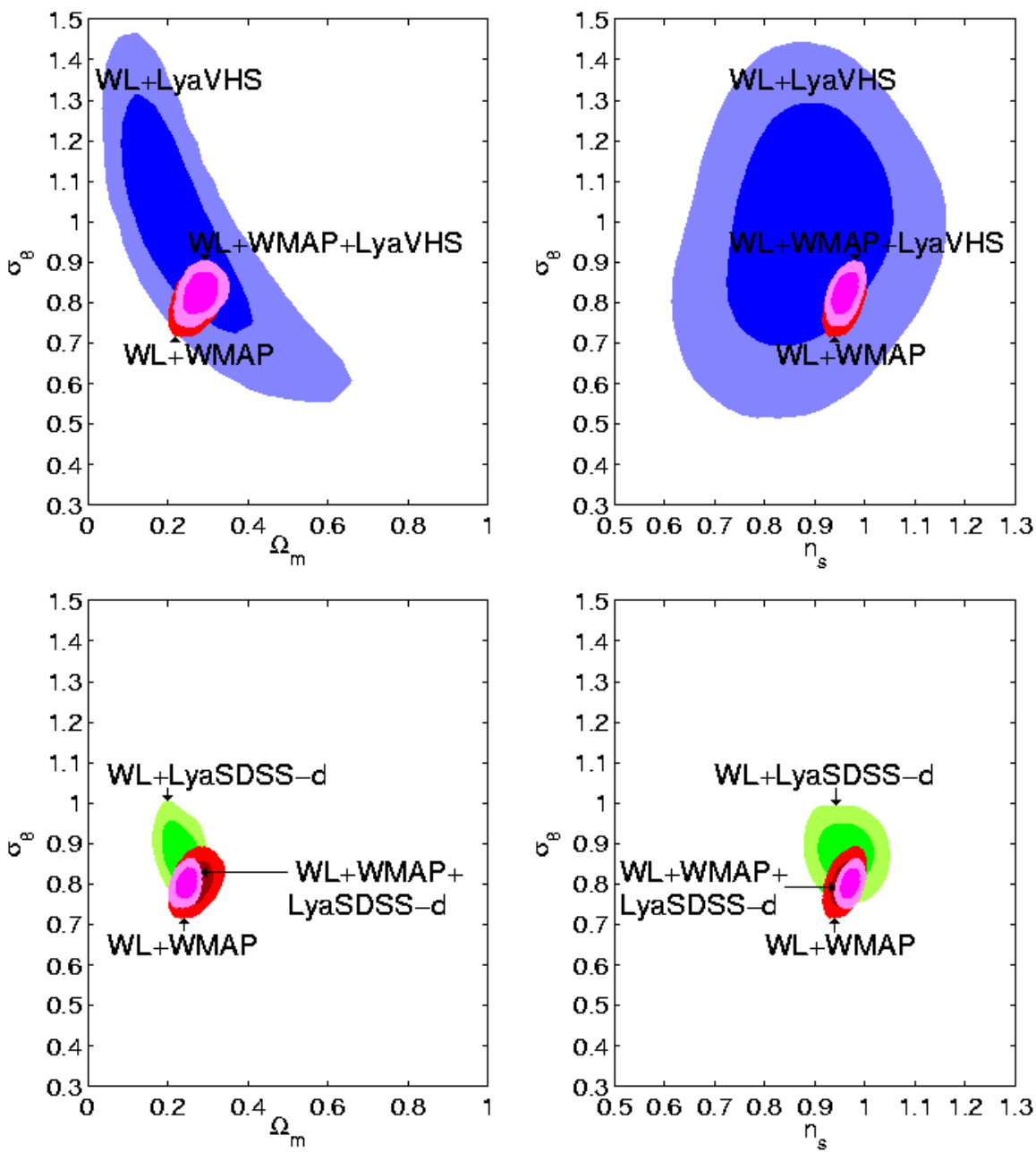

Figure 2. $1-\sigma$ and $2-\sigma$ contours of the marginalized likelihood in the $\sigma_{8}-\Omega_{0 \mathrm{~m}}$ plane (left column) and $\sigma_{8}-n_{\mathrm{s}}$ plane (right column) for various combinations of data sets from COSMOS (WL), WMAP, and Ly $\alpha$ forest data from VHS [16] (Lya VHS, upper panels) and SDSS as analyzed by [15] (Lya SDSS-d, lower panels).

results in a reduction of power on small scale. Small scale experiments like Ly $\alpha$ forest and weak lensing observations are obviously crucial for the determination of a possible running of the spectral index, since they increase the lever arm for primordial spectrum reconstruction. The high $\sigma_{8}$ value preferred by WL and Ly $\alpha$ data excludes the most negative values of $n_{\text {run }}$ found by WMAP, and reduce its error by a factor of two. We find $n_{\text {run }}=-0.028 \pm 0.018$ for WMAP + COSMOS + Ly $\alpha$ VHS and $n_{\text {run }}=-0.007 \pm 0.021$ for WMAP + COSMOS + Ly $\alpha$ SDSS-d (68\% C.L.).

\section{Conclusions}

We have presented a joint analysis of the constraints on the matter power spectrum and the density of dark matter from three different cosmological probes: the CMB temperature and polarization anisotropy measurements of WMAP year three, the state-of-the-art 
A combined analysis of 3D weak lensing, Lyman- $\alpha$ forest and WMAP year three data

Table 4. Same as table 3 for the case with a running spectral index. The quoted values are the mean and $68 \%$ confidence limits.

\begin{tabular}{lll}
\hline & WL + WMAP3 + Ly $\alpha$ VHS & WL + WMAP3 + Ly $\alpha$ SDSS-d \\
\hline$\sigma_{8}$ & $0.809 \pm 0.041$ & $0.818 \pm 0.024$ \\
$n_{\mathrm{s}}$ & $0.965 \pm 0.018$ & $0.971 \pm 0.015$ \\
$\Omega_{0 \mathrm{~m}}$ & $0.304 \pm 0.032$ & $0.255 \pm 0.018$ \\
$h$ & $0.679 \pm 0.026$ & $0.719 \pm 0.018$ \\
$\tau$ & $0.085 \pm 0.037$ & $0.135 \pm 0.026$ \\
$n_{\text {run }}$ & $-0.028 \pm 0.018$ & $-0.007 \pm 0.021$ \\
\hline
\end{tabular}

weak lensing COSMOS survey and two independent Ly $\alpha$ forest data sets. The different observables are prone to very different systematic errors and parameter degeneracies, and more importantly probe different scales and redshifts. Assessing their consistency is an important test of the $\Lambda \mathrm{CDM}$ paradigm and is crucial for further improving constraints on cosmological parameters. The measurements of the matter power spectrum on small and intermediate scales based on Ly $\alpha$ forest and weak lensing data agree very well and suggest a higher amplitude $\left(\sigma_{8}=0.876 \pm 0.048\right.$ with the analysis of SDSS Ly $\alpha$ forest data based on the flux derivatives method of [15]) than the WMAP data alone $\left(\sigma_{8}=0.762 \pm 0.046\right)$. The direction of degeneracy between the amplitude of the power spectrum on galaxy scales and the parameters governing its shape (in other words, the direction of degeneracy in $\sigma_{8}-n_{\mathrm{s}}$ and $\sigma_{8}-\Omega_{0 \mathrm{~m}}$ space) is different for the Ly $\alpha$ forest and weak lensing data. These two observables thus complement each other very well and combining them results in a significant improvement. Combining all three observables we get either $\sigma_{8}=0.800 \pm 0.023$, $n_{\mathrm{s}}=0.971 \pm 0.011, \Omega_{0 \mathrm{~m}}=0.247 \pm 0.016$ (with the analysis of SDSS Ly $\alpha$ forest data based on the flux derivatives method of [15]) or $\sigma_{8}=0.822 \pm 0.032, n_{\mathrm{s}}=0.960 \pm 0.016$, $\Omega_{0 \mathrm{~m}}=0.282 \pm 0.026$ (with the high-resolution Ly $\alpha$ data of VHS). We further explored the constraints for a running of the spectral index and found the data to be consistent with no running at less than the $2 \sigma$ level. Adding the smaller scale data sets reduces the uncertainty on the running of the spectral index by a factor of two with respect to WMAP alone.

\section{Acknowledgments}

Computations were done at the UK National Cosmology Supercomputer Center funded by PPARC, HEFCE and Silicon Graphics/Cray Research and at the HPCF (Cambridge High Performance Computer Cluster), as well as on the MUST cluster in LAPP, Annecy (CNRS \& Université de Savoie). JL acknowledges partial support from the EU 6th Framework Marie Curie Research and Training network 'UniverseNet' (MRTN-CT-2006-035863).

\section{References}

[1] Beltran M, Garcia-Bellido J, Lesgourgues J and Viel M, 2005 Phys. Rev. D 72103515 [SPIRES] [astro-ph/0509209]

[2] Croft R A C et al, 2002 Astrophys. J. 58120 [SPIRES] [astro-ph/0012324]

[3] Gnedin N Y and Hamilton A J S, 2002 Mon. Not. R. Astron. Soc. 334107 [astro-ph/0111194]

[4] Gnedin N Y and Hui L, 1998 Mon. Not. R. Astron. Soc. 29644 [astro-ph/9706219]

[5] Heymans C et al, 2006 Mon. Not. R. Astron. Soc. 3681323

[6] Massey R et al, 2007 Mon. Not. R. Astron. Soc. 37613 
A combined analysis of 3D weak lensing, Lyman- $\alpha$ forest and WMAP year three data

[7] Hinshaw G et al, 2006 Preprint astro-ph/0603451

[8] Jena T et al, 2005 Mon. Not. R. Astron. Soc. 36170 [astro-ph/0412557]

[9] Kim T S, Viel M, Haehnelt M G, Carswell R F and Cristiani S, 2004 Mon. Not. R. Astron. Soc. 347355 [astro-ph/0308103]

[10] McDonald P et al, 2006 Astrophys. J. Suppl. 16380 [astro-ph/0405013]

[11] Page L et al, 2006 Preprint astro-ph/0603450

[12] Spergel D N et al, 2006 Preprint astro-ph/0603449

[13] Springel V, 2005 Mon. Not. R. Astron. Soc. 3641105 [astro-ph/0505010]

[14] Springel V, Yoshida N and White S D M, 2001 New Astron. 679 [astro-ph/0003162]

[15] Viel M and Haehnelt M G, 2006 Mon. Not. R. Astron. Soc. 365231 [astro-ph/0508177]

[16] Viel M, Haehnelt M G and Springel V, 2004 Mon. Not. R. Astron. Soc. 354684 [astro-ph/0404600]

[17] Viel M, Haehnelt M G and Springel V, 2006 Mon. Not. R. Astron. Soc. 3671655 [astro-ph/0504641]

[18] Viel M, Lesgourgues J, Haehnelt M G, Matarrese S and Riotto A, 2005 Phys. Rev. D 71063534 [SPIRES] [astro-ph/0501562]

[19] Viel M, Weller J and Haehnelt M, 2004 Mon. Not. R. Astron. Soc. 355 L23 [astro-ph/0407294]

[20] Zaldarriaga M, Scoccimarro R and Hui L, 2003 Astrophys. J. 5901 [SPIRES] [astro-ph/0111230]

[21] Lewis A and Bridle S, 2002 Phys. Rev. D 66103511 [SPIRES] http://www.cosmologist.info

[22] Seljak U, Slosar A and McDonald P, 2006 J. Cosmol. Astropart. Phys. JCAP10(2006)014 [SPIRES] [astro-ph/0604335]

[23] Seljak U, Makarov A, McDonald P and Trac H, 2006 Phys. Rev. Lett. 97191303 [SPIRES] [astro-ph/0602430]

[24] Viel M, Lesgourgues J, Haehnelt M G, Matarrese S and Riotto A, 2006 Phys. Rev. Lett. 97071301 [SPIRES] [astro-ph/0605706]

[25] Heavens A, Kitching T and Taylor A, 2006 Preprint astro-ph/0606568

[26] Leauthaud A et al, 2007 Preprint astro-ph/0702359

[27] Massey R et al, 2007 Astrophys. J. Suppl. 172239 [astro-ph/0701480]

[28] Massey R et al, 2007 Nature 445286 [SPIRES]

[29] Mobasher B et al, 2007 Astrophys. J. Suppl. 172117

[30] Smith R E et al, 2003 Mon. Not. R. Astron. Soc. 3411131 [astro-ph/0207664]

[31] Lewis A, Challinor A and Lasenby A, 2000 Astrophys. J. 538473 [SPIRES] [astro-ph/9911177]

[32] Rudd D H, Zentner A R and Kravtsov A V, 2007 Preprint astro-ph/0703741

[33] Viel M, Haehnelt M G and Lewis A, 2006 Mon. Not. R. Astron. Soc. 370 51L [astro-ph/0604310]

[34] Bergeron J et al, The large programme 'cosmic evolution of the IGM', 2004 The Messenger 11840

[35] Bardeen J M, Bond J R, Kaiser N and Szalay A S, 1986 Astrophys. J. 30415 [SPIRES] 